\title{
Identification of modal properties of composite thin plate using OMA in wind tunnel environment
}

\begin{abstract}
Identification of modal parameters is crucial especially in aerospace applications whereby the interactions of airflow with aircraft structures can result in undesirable structural deformations. This structural deformation can be predicted with knowledge of the modal parameters. This can be achieved through conventional modal testing that requires a known excitation force in order to extract these dynamic properties. This technique can be experimentally complex because of the need for artificial excitation and it also does not represent actual operational condition. The current work presents part of research work that address the practical implementation of operational modal analysis (OMA) applied to a cantilevered hybrid composite plate exposed to low speed airflow in a wind tunnel. A single contactless sensing system via a laser vibrometer is employed to measure the response. OMA technique applied in a wind-on condition succeeded in extracting the modal parameters of the hybrid composite plate which correlate well with modal testing using impact hammer excitation.
\end{abstract}

Keyword: Hybrid kevlar composite; Laser vibrometer; Modal parameters; Operational modal analysis; Wind tunnel 